\title{
Re-engineering of Human Resources as the Foundation for the New Economy
}

\author{
Andrey Blinov \\ Financial University under the Government of the Russian \\ Federation \\ Moscow, Russia \\ e-mail: aoblinov@mail.ru
}

\author{
Olga Rudakova \\ Financial University under the Government of the Russian \\ Federation \\ Moscow, Russia \\ e-mail: olrud@yandex.ru
}

\begin{abstract}
The article concentrates on the concept of reengineering business processes, which incorporates a largeyield potential for reformation and modernization of various economic systems. It analyzes peculiarities of the current stage development of reengineering based on a cognitive approach. It also covers the importance of the creative potential of the employees as a factor for increased efficiency.
\end{abstract}

Keywords-creative potential; cognitive science; businessprocess; the system approach; cognitive reengineering; cognitive management; reengineering; self-learning organization; creativity.

\section{INTRODUCTION}

It is envisaged that by 2030-2035 half of Russia's GDP will be formed on the basis of smart products, experience economy, education, science and culture, as this is the trend of developed countries in the XXI century. The new economy stipulates investment in human capital for the purpose of increasing returns. A McKinsey survey revealed that as much as $70 \%$ of the corporate M\&A failures stem from human behavior, since the mere modification of business-processes, structure and strategic goals is insufficient. It is of paramount importance to revise the essence of the staff day-to-day working environment. Success (or failure) of any organization depends not only on the quality of goods manufactured and services rendered, but also on the speed at which innovation is applied. Modern organizations require new ideas, approaches, viewpoints and hence - open-minded people ready to embark on that.

Creative, imaginative, inquisitive and not-easy-tomanage person is the key component of the efficient manufacturing process. Up to $40 \%$ of the GDP in developed countries is generated through the work of creative staff [4]. Proper application of skills, talents and perks of talented staff members enables organizations to boost innovation and therefore increase the quality of services/products. Creative potential of employees becomes the major pulling power for the successful development of an organization; efficiency is the pillar and stairway to the achievement of strategic goals.

\section{DeVElopment of THE CREATIVE Potential OF THE EMPLOYEES}

Re-engineering the business processes within the organization is based on the use and development of the creative potential of the employees. To ensure that the staff is able to contribute to the aims of the organization while also being held accountable for the results, it is important to understand the degree of creativity applied and thus to have a tool to measure that. Evaluation itself will become possible only when the working environment meets the necessary conditions.

Employee creativity is defined as the ability and determination of the staff to meet challenges and achieve business goals. One generally defines two basic approaches: motivated and non-motivated. Within the motivated approach, creativity reclines against knowledge, choice of methods, social criteria, culture etc. In the second case, knowledge may not be held as a key factor and thus creativity comes through intuitional actions through trial and error methods.

When solving a particular business case, one may meet with a contradiction between the necessities of the reengineering and actual availability of resources. On the one hand, this drives the internal creativity of each of the employees, and on the other hand, it propels a holistic approach within the whole organization.

Reliance on creativity can originate through the joint efforts of many people that drive the set up of separated groups of creative professionals in the form of teams - which appears to be the most widespread type of such union. Joint cooperation should provide for proper application of skills and perks of a single employee, realize his/her human potential, be complex and comprehensive, leave room for autonomy, self-education and be properly remunerated.

The atmosphere of the working environment drives effectiveness of an organization based on creativity. Should the latter be healthy, it will boost creativity through trust, open-mindedness and the spirit of competition. Reserved characters, ambiguity and mistrust are the characteristics of the non-efficient organization, which cannot function properly and will most likely fail to develop its employees' human potential.

Team development is done via the coaching of both the top management and the employees, as it helps team members to recollect and become closer, thereby becoming 
capable of duly and proactively meeting challenges and coping with critical situations [2].

A team job is a link between a single employee and an organization. Team initiatives can be viewed as a catalyst for both the personal development of the staff and as a way to achieve corporate goals. Therefore, development of the management team is essential for manpower productivity on both an organizational and individual scale. Gradually, such teams will become the decisive factor in the shaping of an organization's competence and drive its strategic development. Therefore, team set up is an inevitable part of an organization's development.

It should be noted that current systems, models and processes of creativity management vary due to differences in strategic goals of different companies and experience, knowledge and resources of those entities.

Management of creativity can be arranged as a separate business-process or be integrated into the general business processes of an organization. They may be linked to the human resources management, which can be achieved via quality management (knowledge, creativity) or via quantitative measures.

These processes can be positioned as a driving force themselves or as a response, since teams deem to link realization of the strategic goals with individual change.

It is important to underline that a team acts as originator, sponsor as well as implementer of the strategy. The process of modeling, use and development of creativity should encompass all teams and employees, as in contemporary elastic organizations (those that lack middle management and have broad responsibilities of team members) the difference between employees and managers are blurred.

\section{REENGINEERING TEAM}

Any change influences various interested parties, with managers often disregarding the human factor. One generally takes into account the change in product assortment, market conditions and new technologies during the growth, decline or reorganization of an organization. It is assumed by default that employees adapt to these changes. However, such changes may negatively influence the working environment and relationships between team members, which reflects on their professional judgment and behavior and is sometimes omitted by management.

Major shifts lead to a mix of reactions from different people. There exists an efficient equilibrium: 20-60-20 $[1,165]$. It implies that any major shift in strategy breaks down as follows: the shift inspires $20 \%$ of employees, $20 \%$ are generally against any changes and the remaining 60\% become the cornerstone of the success/failure of the undertaking. Many surveys reveal that the lion's share of the changes do not lead to anticipated results, such as capitalization growth, market share, cost reduction or access to new market niches.

There are many reasons of failure. One of the key reasons, however, is the non-acceptance of the change by employees. An organization does not change unless there is a change in the mentality of the staff. By altering the behavior, management generally faces emotional reception based on different aspirations of various people [3].

Our own research in this field drives us to the conclusion that the emotional reaction of employees and their viewpoint are not merely crucial, but are of paramount importance to the success of a change. The measuring of personal attitudes would enable the management to make timely adjustments to strategy to make sure that the ultimate goal is reached.

The re-engineering of business-processes is part of the management innovations that must be carried by the gifted employees and managers. This would enable the organization to proceed with the implementation of its strategy.

The launch of the re-engineering should be commenced after considering the answers to the following questions:

- How can the organization convince employees to the necessity of re-engineering?

- Are the internal environment and general methods of an organization adequate to the nature of the changes undertaken and what exactly should be amended?

- Which new skills and abilities are crucial to ensure that employees can successfully perform the planned enhancements and which type of training is essential?

- To what extent is the corporate structure, business processes and remuneration levels are adequate to the sheer state of the change promoted?

Therefore, it is not the company that is being reengineered - it is the staff. The following roles are generally assigned within re-engineering [5,127]:

- Leader: a senior executive who validates the reengineering and ensures motivation;

- Process manager: a person, answerable for a particular process and its re-engineering;

- Re-engineering team: a group of people (team) that investigates the current status of the existing process, its peculiarities and is involved in its refurbishment and implementation of new rules, procedures etc.;

- Organization committee: a body, consisting of a number of senior executives, who elaborate and determine the general strategy of re-engineering and monitor its implementations step by step;

- Head of operations: a person, who is responsible for coordination of efforts within an organization within re-engineering of various business processes to ensure continuity and synergy between them.

The leader appoints the Process Manager, who in his turn assembles the team that conducts re-engineering with the help and with concurrence from the Head of Operations with support of the Organization committee.

However, it is the Leader who gives green light to the reengineering process and attains its ultimate goals. By being 
a senior executive with sufficient internal authority and by having a clear view or the re-organization, the Leader inspires all employees with ambition. A motivated and dedicated Leader is the cornerstone of the re-engineering process. He also appoints the process mangers, formulates key bullet points of the go-ahead plan and creates the business environment within the Team by serving as a living example of ambition, proactive management and a cognitive approach. The Leader combines team members out of the best employees available and removes those who oppose/make a poor contribution to re-engineering. Therefore, most of the re-engineering failures are a direct result of poor leadership.

The Process Manager is responsible for the reengineering of a single process and is obliged to create a working environment for the Team. He manages resources and removes red-tape as well as tries to communicate and interact with of the process managers whose functional groups may fall within the scope of a given business process.

The Process Manager motivates, inspires and renders support to its team members, as well as acts as initial reviewing authority. On the other hand, he acts as intermediary in case ideas from his team are criticized by other process mangers in order to allow his team members to concentrate on re-engineering.

The actual re-engineering is ultimately carried out by the team, which must generate ideas and transform them into plans (and execute those plans as well). This is the real power that transforms the organization.

A single team can conduct re-engineering only of one process. That is why a number of teams must be set up in case more than 1 process is subject to re-engineering. In order to act efficiently a team must consist of $5-10$ members. $[8,125]$ who are subdivided into two sub-groups: (1) those who perfectly know the process and (2) those who are not involved with it.

Employees from the first sub-group are important to understand the aspects that are subject to change. A Team should be arranged to be self-sufficient in terms of internal management.

To ensure the ultimate success team members must work in a single room space, since re-engineering stipulates vast brainstorming and teamwork - sitting in separate cubicles may hinder the whole process. The team must be capable of working with comprehensive and ambiguous issues, not be afraid to make mistakes and be able to learn from those experiences. Statistics show that each team member must dedicate at least $75 \%$ of his working hours to re-engineering otherwise failure is imminent. All day-to-day activities should be left outside the team-room; all the business ties to internal processes should also be torn asunder in order to fully concentrate on the re-engineering.

A team is supported by an additional group of employees that participate in re-engineering on the part-time basis and execute tasks that are more specialized. These can be specialists with insight into generalized field (IT, HR, PR).
The head of operations has a two-fold objective: the first one is to ensure the flawless operational efficiency of the process managers and the teams; the second one is to coordinate the whole re-engineering process. He also monitors performance of the process managers during reengineering and has the authority to initiate intra-team discussions.

The employees' day-to-day environment is greatly affected by the change in business processes as many small positions become multi-functional within re-engineering, leading the fact that managers start to act as coaches, not monitoring authorities. Employees tend to dedicate more time to client needs, not to pleasing their bosses. Employee values and dedication to work also adjust to the new principles.

All team members constantly broaden their fields of responsibilities and authorities. Though the borderline between different job responsibilities is blurred, all team members will concentrate on their specific part and will not do the same, while retaining understanding of the whole process.

Re-engineering tends to exclude not only counterproductive costs, but also low added-value work that increase satisfaction and remuneration levels. Personal development within a team is not linked to hierarchy but to widening of the field of responsibilities. The old model was based on the principle "simple task for simple people. The new concept is dominated by another motto: comprehensive tasks for creative employees. After the re-engineering, it will be difficult to find a job with merely a simple list of tasks. This raises expectations from the staff; they must show initiative, discipline, motivation and suit the clients' needs.

In the permanently changing environment, it is hard to find people who possess all skills and knowledge. Therefore, constant training of employees is inevitable and should be inherent [6].

Effectiveness of employees and their remuneration are driven by the outcome of their job. In case the job is subdivided into a series of simple tasks, the efficiency of an employee can be determined only based on execution of those tasks. However, the problem is that the increase in the efficiency of execution does not necessarily lead to an increase in efficiency of the project itself. When employees do something as a team the performance can be evaluated by analyzing the benefit they created. The remuneration comes in the form of a bonus, not a salary increase.

Remuneration is becoming less dependent on the hierarchy of the position of an employee or number of subordinates/size of the managed portfolio or time spent "in the office". It is derived from the positive impact on the performance of the whole organization and its efficiency.

Employees tend to refrain from sticking to their position, but effectiveness of the work they do. Re-engineering involves massive transformation of the corporate culture of an organization, not only in its corporate structure. Dominating corporate values should mirror the efficiency of 
the business processes. [7,133]. Employees must be convinced that they work for their clients, not their immediate supervisors, and that should be reflected by the remuneration policy since this is the key factor which transfers corporate values to the staff. It should be understood that the introduction of new processes would fail if these values remain the same.

Contemporary re-engineering differs from the concept of the 1990s as it has been influenced by cognitology, which is defined as the science that covers cognitive processes and consciousness, and the processes of interaction between perception, understanding, modification, imagination and reflection and self-education. Additionally, it includes the modeling of artificial intellectual systems based on the analytical, synthetic and synergy concepts. Informational, cybernetic, systematic and synergetic approaches are the methodological cornerstone of this approach. [2 ].

The development of cognitology is going into such "irrational" fields as intuition and creativity. Business practice shows that there are many situations in which spontaneous decisions are made, and subsequent consideration of such decisions reveals that positive outcomes can be achieved even without thorough consideration and despite a chaotic influx of data.

Understanding of the fact that competition has migrated from competition for natural resources to competition for intellectual resources has led to the evolution of the cognitive management concept.

Cognitive management is a systematic process management that concentrates on the identification of knowledge, accumulation and distribution of data within an organization for efficiency purposes.

Principles of cognitive management are:

- Knowledge originates and rests in the consciousness of people;

- Mutual use and application of knowledge is based on trust;

- Advanced technologies lead to development of new forms of cognitive behavior;

- Knowledge is act of will through creativity and its development should be encouraged [1].

Information technologies assume a new role in the reengineering process: they transform a mere instrument of innovation into the basis for the organization's evolution, because even the most sophisticated IT background will be rendered null and void without a significant change in the employee competence (responsibilities and authorities) and capabilities (skill, perks, etc).

\section{CONCLUSION}

Given the above modern situation, Russian organizations require new methods, which should be elaborated based on a synergy between humanitarian and technocratic approaches. This new approach would be centered on state-of-the-art IT and technological solutions. It is essential to ensure the development of a knowledge management concept alongside a cognitive approach to human behavior since that turns out to be the most sought-after instrument for modernization and innovation and the best tool for ensuring the full-scale use and application of human creative potential.

It is the cognitive re-engineering that meets all of the above requirements. Its key priority is to create a selflearning organization, that will resemble an organization that creates, acquires, transfers and uses knowledge and know-how and is capable of duly reacting and proactively responding to the challenges of the business environment.

\section{REFERENCES}

[1] Abdikeyev N.M., Kiselev A.D. Knowledge management and reengineering the corporation business / Under nauch.red. Dr. Technical Sciences, prof. N.M.Abdikeev. - M .: INFRA-M, 2011.

[2] Blinov A.O., Lisichkina Y.S., Rudakova O.S. Management of organizational changes organizations through cognitive technologies // Economics and Entrepreneurship, \#5 (Part 1), 2015, pp.597-599.

[3] Blinov A.O., Rudakova O.S. Reengineering of human resources as a means of competitive institutions // Economics and Management: Problems and Solutions. \#4 2013 pp. 62-69.

[4] Blinov A.O., Rudakova O.S., Ugryumova N.V. Management of organizational changes at the industrial enterprises // Economy. Taxes. Right, \#1, 2013 pp.45-53

[5] Hammer M., Ciampi J.. Reengineering the Corporation: A Manifesto revolution in business / Michael Hammer, James Champy; per. from English. Yu.E.Kornidovich. - M .: Mann, Ivanov Fereber, 2006.

[6] Milner B.Z. Knowledge management: evolution and revolution in organization- M.: INFRA-M, 2003

[7] Prigogine A.I. Disorganization: Causes, types, bridging / A.I.Prigozhin.- M .: Alpina Business Books, 2007

[8] Robson M., Ullah F. Business Process Reengineering: A Practical Guide / Trans. from English. Ed. N.D.Eriashvili. M .: UNITY-DANA 2003. 